\title{
Fourier Analysis of the Texture of High-Definition Images
}

\author{
Olena Osharovska, Mikola Patlayenko, Volodymyr Pyliavskyi \\ Dept. Radio and Television Broadcasting, \\ line 2: name of organization, acronyms acceptable O.S. Popov Odessa National Academy of Telecommunications, \\ Odessa, Ukraine, 65029 , \\ e-mail: osharovskaya@gmail.com, nick_msa@ukr.net, v.pilyavskiy@ukr.net
}

\begin{abstract}
This paper presents the results of measuring the signal-to-noise ratio at the boundary of object contours in highdefinition images after compression of the original digital stream based on the Fourier transform and the discrete cosine transform. It is shown that when choosing the threshold for limiting the spectral components of the image, one should not only focus on the frequency, but also take into account the magnitude of the differences in the brightness signal in areas of low-contrast textures.
\end{abstract}

Index Terms-Image; texture; Fourier transform; limit threshold; signal-to-noise ratio

\section{INTRODUCTION}

The development of new methods for compressing still images and intra-frame compression of video sequences is a key element in the construction of broadband image transmission systems for various purposes [1, 2]. In this respect, the main criterion for the degree of compression is subjective estimates, and estimates obtained by measuring distortions of sharp image boundaries resulting from spectral processing and compression. For images of high and ultrahigh definition when viewed on large-size screens with high resolution, it becomes possible to distinguish such fine details as composition and material of textures.

\section{SELECTING IMAGES FOR EVALUATION}

The choice of test images was made from considerations of compromise, first, between the possible degree of compression and preservation of textures of images where it is low-contrast, so that the image of diffusely reflecting surfaces does not turn into an image of shiny objects.

Some of the test images are shown in Table. I. In the image called "Kimono", a picture on the clothes of a doll representing small flowers can be viewed as a low-contrast texture. The color components in this low-intensity texture, respectively, the brightness component has insignificant fluctuations. An even more complicated case for compression is the image "Stained glass", in which, on a very dark background of the cathedral, you need to transfer the texture of the stained glass. The third image is easier to analyze because of the larger difference between the background and the texture of the butterfly's wings.

TABLE I. TEST IMAGE WITH A HIGHLIGHTED TEXTURE

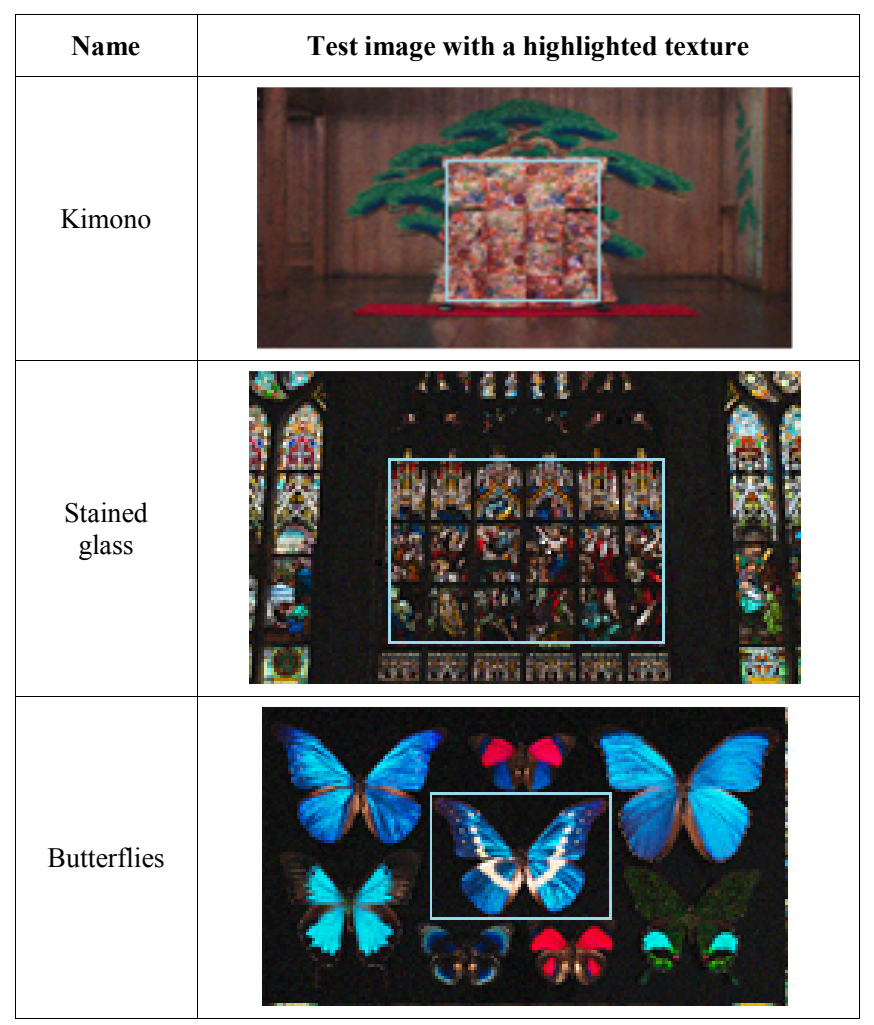

The relative total space of the image areas with high detail in the selected pictures varies from $2 \%$ to $6 \%$, and the relative level of the texture signal change on the border areas was only between $0.3 \%$ and $0.7 \%$.

The second factor for selecting test images was the possibility of free access to image databases [3]. We selected super high definition images with a resolution of $4 \mathrm{~K}$ and the number of decomposition elements $3840 \times 2160$. Since the display we used as a viewing screen was only full $\mathrm{HD}$, the 
original images were subjected to a decimation step 2 both horizontally and vertically. Images are obtained with dimensions of $1920 \times 1080$.

\section{FOURIER TRANSFORM WITH COEFFICIENT CONSTRAINT}

The classical notion of the image spectrum $F\left(v_{H}, v_{V}\right)$ corresponds to a two-dimensional discrete Fourier transform (1).

$$
F\left(v_{H}, v_{V}\right)=\sum_{x=0}^{N_{x}-1} \sum_{y=0}^{N_{y}-1} B(x, y) e^{-j 2 \pi\left(\frac{x v_{H}}{N_{x}}+\frac{y v_{V}}{N_{y}}\right)},
$$

where:

- $B(x, y)$ is the value of the brightness (or color difference) signal of the image element with the coordinates $(x, y)$;

- coordinates are represented by integers and do not exceed the maximum size of the image horizontally $N x$ and the vertical $N y$;

- $v_{H}, v_{V}$ are space frequencies in horizontal and vertical directions.

In the Matlab environment, we perform Fast Fourier transform of the whole image, we get $1920 \times 1080$ coefficients, and find the module for each component. We apply a restriction on the level, replacing the spectral components that are less than a given threshold by zero values. The thresholds used the percentage value of the maximum brightness of the signal within the selected texture. The calculated values of the signal-to-noise ratio near the contours are shown in Table II for four variants of thresholds.

TABLE II.

QUALITY INDICATORS FOR FOURIER TRANSFORMATION

\begin{tabular}{|l|c|c|c|c|}
\hline \multirow{2}{*}{ Test Image } & \multicolumn{4}{|c|}{ EPSNR, dB } \\
\cline { 2 - 5 } & $0.5 \%$ & $1 \%$ & $2 \%$ & $5 \%$ \\
\hline Kimono & 26.4 & 24 & 23.3 & 20.7 \\
\hline Stained glass & 24.5 & 23 & 21.3 & 18.8 \\
\hline Butterflies & 22.4 & 21 & 19.2 & 18.6 \\
\hline
\end{tabular}

Analysis of the results of Table II suggests that the DFT does not provide good quality of the image as a result of limiting the spectral components. The low-contrast textures simply disappear. The qualitative parameters of the image also depend on the bit of the representation of the luminance and chroma signals. Table III presents the qualitative indices and the compression ratio $\mathrm{K}$ for two test images for word lengths varying from six to twelve.

TABLE III. DEPENDENCE OF EPSNR AND K ON THE WORD LENGTHS

\begin{tabular}{|c|c|c|c|c|c|c|}
\hline Bit & EPSNR & PSNR & K & EPSNR & PSNR & K \\
\hline \multicolumn{3}{|c|}{ Kimono } & \multicolumn{3}{c|}{ Stained glass } \\
\hline 6 & 36.0 & 43.8 & 24 & 33.5 & 42.8 & 29 \\
\hline 8 & 37.7 & 45.8 & 21 & 34.5 & 44.1 & 27 \\
\hline 10 & 39.2 & 47.6 & 18 & 35.3 & 45.1 & 26 \\
\hline 12 & 40.6 & 49.3 & 16 & 36.1 & 46.0 & 23 \\
\hline
\end{tabular}

As can be seen from the results, as the word length increases, the quality indicators improve, but the compression ratio decreases.

For the test image "Butterfly", the dependence of word length and PSNR is presented in Fig. 1.

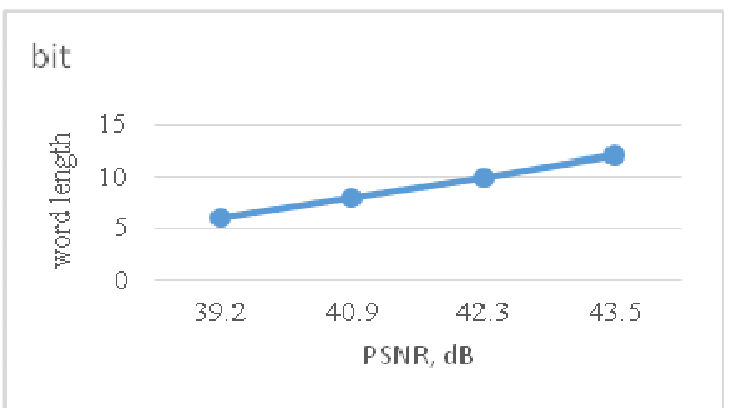

Figure 1. Dependence of the PSNR on the number of bit for "Butterfly"

The dependence of EPSNR on the compression ratio for the Butterfly is shown in Fig. 2.

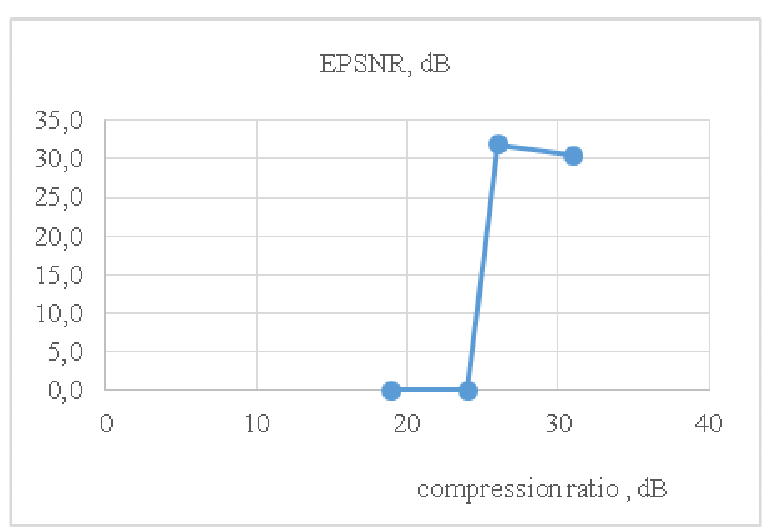

Figure 2. Dependence of the EPSNR from compression ratio for "Butterfly"

EPSNR dependence on the compression ratio has a pronounced optimum, after which the image quality begins to drop with higher compression ratio.

\section{DISCRETE COSINE TRANSFORMATION AND CONSTRAINT OF COEFFICIENTS}

The JPEG and MPEG-2 standards use DCT [5-8]. A feature is segmentation in blocks raw image data is divided into blocks of $8 \times 8$ pixels (these blocks are the minimum coded block). This means that the JPEG compression algorithm largely depends on the position and alignment of the boundaries of these blocks. In MPEG-4, the block sizes vary from $4 \times 4$ up to $16 \times 16$.

Given the values obtained in the DCT step (2), they are sorted in order of low-frequency components (changes that occur at a greater distance from the image block) to highfrequency components (changes that can occur with each pixel). It is widely known that people are more critical of errors in low-frequency information than high-frequency information. The JPEG algorithm discards many of these highfrequency (noise-like) details and saves slowly changing 
information about the image. This is done by dividing all the coefficients of the equation by the corresponding value in the quantization table and rounding the result to the nearest integer (4). Components that had either a small coefficient or a large divisor in the quantization table are likely to be rounded to zero. The lower the quality setting, the greater the divisor, which gives a greater chance of getting a zero result. On the other hand, the highest quality setting would have the values of the quantization table of all 1 , which means that all the original DCT data is stored.

$$
F\left(v_{H}, v_{V}\right)=\frac{1}{4} C_{v_{H}} C_{v_{V}} \sum_{x=0}^{N_{x}-1} \sum_{y=0}^{N_{y}-1} B(x, y) \times S,
$$

where the kernel $S$ of the transformation is (3):

$$
\begin{gathered}
S=\frac{\cos \left((2 x+1) \pi v_{H}\right) \cdot \cos \left((2 y+1) \pi v_{V}\right) .}{2 N}, \\
C_{v_{H}} C_{v_{V}}=\left(\begin{array}{c}
1 / \sqrt{2}-\text { for_v } v_{H}, v_{V}=0, \\
0_{-} \text {otherwise }
\end{array}\right. \\
F_{q}\left(v_{H}, v_{V}\right)=\text { round }\left(\frac{F\left(v_{H}, v_{V}\right)}{Q_{v_{H}, v_{V}}}\right),
\end{gathered}
$$

The values of the matrices of the quantization coefficients for the luminance component each firm offers its own. For example, the quantization matrix for the SONY DSC-N2 (fine) camera is shown in (4). The spectrum obtained from the quantization and rounding in the DCT is shown in Fig. 3.

$$
Q_{v_{H}, v_{V}}=\left[\begin{array}{llllllll}
1 & 1 & 1 & 1 & 1 & 2 & 3 & 4 \\
1 & 1 & 1 & 1 & 2 & 3 & 4 & 3 \\
1 & 1 & 1 & 1 & 2 & 3 & 4 & 3 \\
1 & 1 & 1 & 2 & 3 & 5 & 5 & 4 \\
1 & 1 & 2 & 3 & 4 & 7 & 6 & 5 \\
1 & 2 & 3 & 4 & 5 & 6 & 7 & 6 \\
3 & 4 & 5 & 5 & 6 & 7 & 7 & 6 \\
4 & 6 & 6 & 6 & 7 & 6 & 6 & 6
\end{array}\right] .
$$

\begin{tabular}{|c|c|c|}
\hline \multirow{2}{*}{ Quantization table } & Kimono & Butterflies \\
\hline & EPSNR, $d B$ & EPSNR, $d B$ \\
\hline SONY - DSC-N2 (fine) & 46.2 & 45.1 \\
\hline Canon PowerShot A700 (superfine) & 45.4 & 48.2 \\
\hline NIKON D80 (FINE) & 44.9 & 47.4 \\
\hline NIKON - COOLPIX S10 (FINE) & 48.2 & 46.8 \\
\hline NIKON - E8800 (EXTRA) & 46.9 & 45.5 \\
\hline JPEG standard & 43.6 & 48.4 \\
\hline
\end{tabular}

Table IV shows the EPSNR for the quantization matrices of different cameras.

TABLE IV.

DEPENDENCE OF EPSNR AND K ON THE WORD

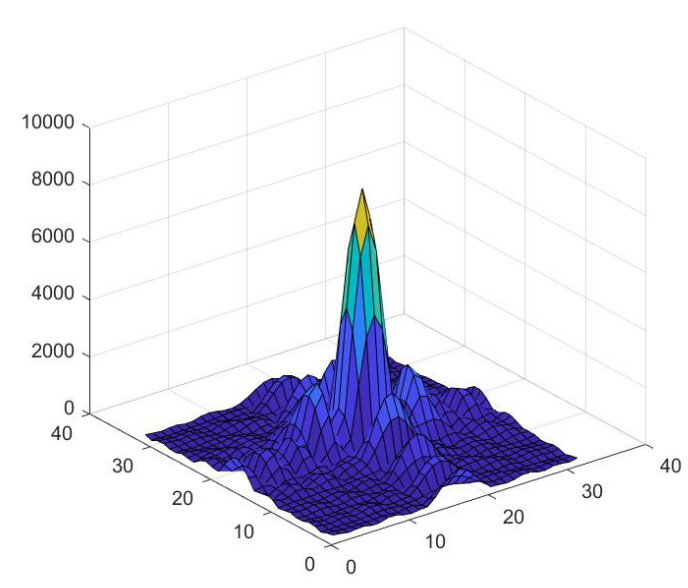

Figure 3. The spectrum o for the SONY DSC-N2 (fine)

As can be judged from Fig. 3, the high-frequency components are preserved, but in comparison with the lowfrequency components, they have a relatively small level

\section{CONCLUSION}

Visual quality assessment method based on the visibility of error between original and distorted images, made it possible to investigate the influence of frequency-dependent quantization of spectral components on the quality of the reproduction of textures. Criteria are formulated for the choice of quantization matrices that provide subjectively correct reproduction of high-frequency components of fine-textured textures.

\section{REFERENCES}

[1] Recommendation ITU-R BT.709-6: 2015, Parameter values for the HDTV standards for production and international programme exchange.IV

[2] Recommendation ITU-T T.81 :1992 Information technology - Digital compression and coding of continuous-tone still images - Requirements and guidelines.

[3] Adobe image data base. Internet resource: https://stock.adobe.com/premium.

[4] Oleg Gofaizen, Olena Osharovska, Mikola Patlayenko, Volodymyr Pyliavskyi, "Test signals for assessment image quality in HD and UHD TV video path", 2016 8th International Conference on Ultrawideband and Ultrashort Impulse Signals (UWBUSIS), pp. 42-46, 5-11 Sept., 2016, DOI: 10.1109/UWBUSIS.2016.7724147.

[5] Recommendation ITU-T J.144:2004, Recommendation BT,1908:2012, "Objective perceptual video quality measurement techniques for digital cable television in the presence of a full reference".

[6] Recommendation ITU-T J.246:2008, Perceptual visual quality measurement techniques for multimedia services over digital cable television networks in the presence of a reduced bandwidth reference.

[7] Recommendation ITU-T J.247: 2008,Objective perceptual multimedia video quality measurement in the presence of a full reference.

[8] International Standard ISO/IEC 15444-1 :2016 | Recommendation ITUT T.800:2015 | Recommendation ITU-T T.800: 2015, Information technology - JPEG 2000 image coding system: Core coding system. 